\title{
Procédure et information des parents lors de l'introduction de nouveaux tests de dépistage néonatal
}

Le 19 novembre 2010, l'Académie Suisse des Sciences Médicales (ASSM) avait organisé une réunion d'experts sur le thème du «Dépistage néonatal: L'évolution technologique, la santé publique et l'autonomie des parents». Partant de l'introduction du dépistage néonatal de la fibrose kystique (FC, mucoviscidose) en Suisse, les participants ont eu l'occasion de faire le point sur la pratique actuelle et les conditions cadres juridiques et éthiques requises lors de l'introduction et de la réalisation de tests de dépistage.

\section{L'introduction de nouveaux tests de dépis- tage selon I'exemple du dépistage FC}

L'initiative pour l'introduction du dépistage FC émanait d'experts médicaux (Swiss Working Group for Cystic Fibrosis, SBGCF). Dans une première phase, tage systématique est réglementée par l'article 12 de la Loi fédérale sur l'analyse génétique humaine (LAGH) et est soumise à autorisation. Dans le cadre du programme du dépistage exigé par l'article 12 LAGH, le SBGCF a démontré à l'Office fédérale de la santé publique (OFSP) que l'évidence scientifique nécessaire à l'introduction du dépistage (basé sur la littérature internationale) existait bien et que les structures organisationnelles indispensables à sa réalisation pouvaient être garanties (best available evidence about clinical and social implication).

Fin 2010, l'OFSP avait donné son accord pour une phase pilote de deux ans débutant le $1^{\text {er }}$ janvier 2011; le programme est à présent concrétisé (déroulement, information des parents, etc.) et les modalités du monitoring sont fixées. Le déroulement de la phase

\section{Pour le financement après la phase pilote, une prise en charge des frais des examens de dépistage par l'assurance obligatoire des soins est visée.}

Correspondance: SAMW/ASSM lic. iur. Michelle Salathé Petersplatz 13 CH-4051 Bâle

m.salathe@samw.ch l'évaluation des dommages et des bénéfices du dépistage FC constituait une priorité. A cet égard, les critères de Wilson et Jungner ont été appliqués:

- La maladie à mettre en évidence constitue-t-elle un problème de santé majeur?

- L'évolution naturelle de la maladie est-elle connue?

- Existe-t-il un stade identifiable ou latent?

- Existe-t-il un traitement efficace et acceptable?

- Existe-t-il des structures pour le dépistage précoce et le traitement de la maladie?

- Existe-t-il une méthode de dépistage adaptée?

- La méthode de dépistage est-elle acceptable pour la population et les experts?

- Existe-t-il un consensus sur les critères de la maladie?

- Existe-t-il un programme de dépistage établi?

- Les coûts du dépistage et de la thérapie sont-ils acceptables vis-à-vis des dépenses globales?

Le dépistage de la FC constitue un examen génétique. La réalisation de l'analyse dans le cadre d'un dépis- pilote est financé par des fonds privés; l'évaluation exigée par l'OFSP sera financée par le mandant.

Pour obtenir l'autorisation définitive pour le dépistage FC à la fin de la phase pilote de deux ans, il faudra une nouvelle fois démontrer à l'OFSP que les conditions requises dans l'article 12, al. 2 LAGH sont toujours remplies, notamment que le bénéfice dépasse clairement les dommages possibles et que la direction du programme (y inclus le conseil aux parents) et le monitoring (l'évaluation) du dépistage sont garantis. L'OFSP décide de délivrer l'autorisation après avoir auditionné la Commission d'experts pour l'analyse génétique humaine (GUMEK) et, si nécessaire, la Commission Nationale d'Ethique dans le domaine de la médecine humaine (CNE). La GUMEK a publié une recommandation dans laquelle sont détaillées les conditions requises aux demandes pour effectuer des dépistages selon l'article 12 LAGH [1].

Pour le financement après la phase pilote, une prise en charge des frais des examens de dépistage par l'assurance obligatoire des soins conformément à la Loi fédérale sur l'assurance-maladie (LAMal) est visée 
(élargissement de l'actuel dépistage néonatal). Sur la base de requêtes documentées et de la recommandation de la Commission fédérale des prestations générales et des principes (CFPP) et de la Commission fédérale des analyses, moyens et appareils (CFAMA) [2], cette décision incombe au Département de l'intérieur. L'OFSP assure le secrétariat de ces commissions et prépare les consultations.

\section{Information des parents concernant les procédures de dépistage néonatal}

Lors de la réunion d'experts, l'exemple du test de Guthrie a démontré que l'information des parents au sujet du dépistage néonatal est actuellement très divergente. Bien qu'il existe une brochure d'information générale, certaines institutions distribuent leur propre documentation interne.

\section{Recommandations}

Les points suivants ont recueilli l'unanimité des participants:

- L'introduction d'un nouveau test de dépistage est chère et les procédures et compétences sont confuses et trop peu connues.

- Les procédures au sein de l'OFSP (évaluation par l'organe d'exécution de la loi avec implication de la GUMEK, CFPP, CFAMA) pourraient être mieux coordonnées, en particulier en ce qui concerne les documents à fournir et la concertation des différents services impliqués.

- L'information des parents devrait être améliorée. Suite à la réunion d'experts, l'ASSM formule les recommandations suivantes:

\section{A l'attention de l'OFSP}

- Sur la base des expériences réalisées avec le premier projet concret de test de dépistage des maladies génétiques, les procédures d'introduction d'un nouveau test de dépistage devraient être coordonnées et simplifiées au sein de l'OFSP. Les spécialistes qui projettent l'introduction d'un nouveau test devraient être soutenus pendant toute la phase de préparation. Cette tâche pourrait éventuellement être assumée par un institut national de prévention ou une institution similaire, tel que le prévoit le projet de loi sur la prévention.
- Il serait judicieux de réglementer la procédure d'autorisation pour le test de dépistage de maladies non génétiques (par ex. infections), qui pourront éventuellement être centralisées dans le centre de dépistage actuel.

\section{A l'attention du centre de dépistage et \\ des sociétés de disciplines médicales}

- Les parents doivent être informés de l'offre de dépistage par différents canaux (gynécologue, sagefemme, hôpital) et à divers moments (avant et après la naissance), à l'aide d'une brochure d'information au format uniforme pour toute la Suisse (lui permettant de bénéficier d'une reconnaissance élevée); celle-ci peut, en fonction des besoins des parents, être complétée par des informations orales. Il va de soi que le consentement des parents est une exigence légale et éthique; la brochure doit préciser que la participation à un test de dépistage est tout à fait libre. Le dépistage néonatal (Guthrie avec dépistage FC) ne requiert toutefois pas - comme jusqu'à ce jour - le consentement écrit des parents; un accord oral est suffisant.

- Les professionnels de la santé devraient être préparés à mener des entretiens; à cet égard l'élaboration d'une brochure spécialisée est tout particulièrement conseillée. Le centre de dépistage met à la disposition des professionnels de la santé (sages-femmes, etc.) les informations et le matériel d'enseignement nécessaires et nomme des interlocuteurs qui se tiennent à disposition pour les questions plus pointues.

\section{Références}

1 Voir: Recommandation 4/2009 concernant les conditions requises aux demandes pour effectuer des dépistages selon l'art. 12 LAGH. www.bag.admin.ch/ themen/medizin/00683/02724/04638/05559/index. html?lang=fr

2 Voir: Manuel pour la présentation de demandes de prise en charge par l'assurance de prestations nouvelles ou controversées et le formulaire de demande et les explications sous: www.bag.admin.ch/themen/krankenversicherung/ 00263/00264/04853/index.html?lang=fr 